\title{
Outsourcing Secure Two-Party Computation as a Black Box
}

\author{
Henry Carter \\ Georgia Institute of Technology \\ carterh@gatech.edu
}

\author{
Benjamin Mood \\ University of Florida \\ bmood@ufl.edu
}

\author{
Patrick Traynor \\ University of Florida \\ traynor@cise.ufl.edu
}

\author{
Kevin Butler \\ University of Florida \\ butler@cise.ufl.edu
}

\begin{abstract}
Secure multiparty computation (SMC) offers a technique to preserve functionality and data privacy in mobile applications. Current protocols that make this costly cryptographic construction feasible on mobile devices securely outsource the bulk of the computation to a cloud provider. However, these outsourcing techniques are built on specific secure computation assumptions and tools, and applying new SMC ideas to the outsourced setting requires the protocols to be completely rebuilt and proven secure. In this work, we develop a generic technique for lifting any secure two-party computation protocol into an outsourced two-party SMC protocol. By augmenting the function being evaluated with auxiliary consistency checks and input values, we can create an outsourced protocol with low overhead cost. Our implementation and evaluation show that in the best case, our outsourcing additions execute within the confidence intervals of two servers running the same computation, and consume approximately the same bandwidth. In addition, the mobile device itself uses minimal bandwidth over a single round of communication. This work demonstrates that efficient outsourcing is possible with any underlying SMC scheme, and provides an outsourcing protocol that is efficient and directly applicable to current and future SMC techniques.
\end{abstract}

\section{Introduction}

As the mobile computing market continues to grow, an increasing number of mobile applications are requiring users to provide personal or context-sensitive information. However, as the recent iCloud breach demonstrates [28], these application servers cannot necessarily be trusted to maintain the security of the data they possess. To better preserve privacy and the functionality of mobile applications, secure multiparty computation (SMC) techniques offer protocols that allow application servers to process user data while it remains encrypted. Unfortunately, while a plethora of SMC techniques exist, they currently require too much processing power and device memory to be practical on the mobile platform. Furthermore, the bandwidth and power requirements for these SMC protocols will always be a limiting requirement for mobile applications even as the computational resources of mobile devices grow.

To bring SMC to the mobile platform in a more efficient way, recent work has focused on developing secure techniques for outsourcing the most expensive computation. Rather than naively trusting the Cloud to stand in for the mobile device in a standard SMC protocol, these outsourced protocols seek to use the Cloud for computation without revealing any input or output values. A number of these protocols have been specifically developed to outsource garbled circuit protocols [27, 8, 7]. These protocols attempt to optimize the outsourcing operations without increasing the complexity of the circuit being evaluated. However, because of this optimization goal, they are constructed and proven secure using specific garbled circuit evaluation techniques. As new techniques for SMC are developed that modify the garbled circuit construction (or use completely different underlying constructions), it is unclear whether these specific outsourcing protocols will be able to take advantage of the new developments.

In this work, we develop a technique for outsourcing secure two-party computation for any two-party SMC technique. Rather than avoiding changes to the function being evaluated, we add a small amount of overhead to the evaluated function itself. This tradeoff allows for an outsourcing scheme that relies on the underlying two-party protocol in a black-box manner, meaning the underlying protocol can be swapped for any other protocol meeting the same definition of security This makes the task of securely incorporating newly developed SMC techniques trivial. This 
protocol enables mobile devices to participate in any secure two-party SMC protocol with minimal cost to the device and with nominal overhead to the servers running the computation. Specifically, we make the following contributions:

- Develop a black-box outsourcing protocol: We develop a novel outsourcing technique for lifting any twoparty SMC protocol into the two-party outsourced setting. To do this, we add a small amount of overhead to the function being evaluated to ensure that none of the inputs are modified by malicious participants. This technique of augmenting the evaluated circuit has been successfully used in other SMC protocols to balance performance with security guarantees [22, 32, 44]. In addition, we leverage the non-collusion assumption used throughout the related work to produce an output consistency check that incurs trivial overhead. While this approach slightly increases the cost of evaluation, it minimizes the computation and bandwidth required by the mobile device.

- Prove security for any underlying two-party SMC protocol: We provide simulation proofs of security to demonstrate that our protocol is secure in the malicious threat model. The only requirement of the underlying two-party SMC protocol is that it satisfy the canonical ideal/real world simulation definition of security against malicious adversaries [16]. This allows any future SMC protocols that are developed to be used in a plug-\&-play manner with our outsourcing technique.

- Implement and evaluate the overhead cost of the outsourcing operations: Using the garbled circuit twoparty SMC protocol of shelat and Shen [44], we implement our protocol and evaluate the complete overhead cost of outsourcing. Rather than compare to previous outsourcing schemes, we instead measure the overhead incurred by augmenting the desired functionality, as well as the input and output preparation and checking. This measurement of cost better represents the value of the scheme, as a direct comparison to previous outsourcing protocols would drastically change depending on the underlying two-party SMC protocol implemented in our scheme. Our results show that for large circuits, black-box outsourcing incurs negligible overhead (i.e., the confidence intervals for outsourced and server only execution intersect) in evaluation time and in bandwidth required when compared to evaluating the unmodified function. To demonstrate the practical performance of our protocol, we develop a mobile-specific facial recognition application and analyze its performance.

This work extends the results presented by Carter et al. [9]. The rest of this work is organized as follows: Section 2 describes related research, Section 3 outlines definitions of security, Section 4 formally defines the protocol, Section 5 provides an overview of security, Section 6 presents formal security proofs, Section 7 describes our implementation and performance evaluation, Section 8 presents a new mobile-specific application for SMC, Section 9 compares the overhead of our black box technique to previous work, and Section 10 provides concluding remarks.

\section{Related Work}

Since it was initially conceived in the early 1980's [45, 17], secure multiparty computation (SMC) has grown from a theoretical novelty to a potentially useful and practical cryptographic construction. The FairPlay implementation [36] provided one of the first schemes for performing secure multiparty computation in practice. Since then, a number of other protocols and implementations have shown that privacy-preserving computation in the semi-honest threat model can be performed relatively efficiently [21, 4, 1]. However, this security model is weak in practice, and does not provide enough security for most real-world situations. To resolve this, recent study has focused on developing protocols that are secure in the malicious setting. For two-party computation, the garbled circuit construction has seen a large amount of new development [33, 34, 37, 42, 31, 43, 44] that has drastically reduced the cost of circuit checking and the associated consistency verification. Because the cut-\&-choose construction that is typically applied in this setting is very costly, recent work has sought to minimize the cost of the cut-\&-choose [13, 32, 23] or amortize that cost over a batch of circuit executions [35, 24]. Besides the garbled circuit technique, other techniques using somewhat homomorphic encryption [11, 10] and oblivious transfer [40] have shown promise of producing efficient protocols for secure multiparty computation in the malicious threat model. However, all of these techniques still have significant overhead cost that makes them infeasible to execute without sizable computational resources.

With smartphone applications retrieving private user data at an increasing rate, secure multiparty computation could potentially offer a way to maintain privacy and functionality in mobile computing. However, the efficiency challenges of secure multiparty computation are compounded when considered in the resource-constrained mobile environment. Previous work has shown that smartphones are generally limited to simple functions in the semi-honest setting [6, 20]. Demmler et al. [12] showed how to incorporate pre-computation on hardware tokens to improve 
efficiency on mobile devices, but still in the semi-honest setting. In addition to the cost of evaluating these SMC protocols, Mood et al. [39] and Kreuter et al. [30] demonstrated that even with significant optimization, the task of compiling circuits on the mobile device can also be quite costly.

Given these limitations, evaluating SMC protocols directly on mobile hardware does not seem to be possible in the immediate future. Because of this, mobile secure computation research has recently focused on applying techniques from server-assisted cryptography [3] to move the most costly cryptographic operations off of the mobile device and onto a more capable cloud server. To achieve this, many authors have focused on developing protocols for outsourcing secure computation of specific algorithms such as graph algorithms [5], set intersection [29], and linear algebra functions [2]. The first protocol to outsource secure multiparty computation for any function was developed by Kamara et al. [26, 27]. In this work, the authors established a definition of security that assumes specific parties in the computation, while malicious, are not allowed to collude. Following on this definition, several other protocols and efficiency improvements have been developed for the outsourced setting [8, 38, 7]. Unfortunately, all of these protocols are built on specific secure multiparty computation assumptions and techniques. With new and varying techniques for SMC being developed at a rapid pace, it is unclear how to apply the outsourcing techniques used in these protocols to new schemes to allow them to benefit from new efficiency improvements. In this work, we seek to develop a protocol that can lift any two-party SMC protocol into the outsourced setting with little overhead.

In recent work, Jakobsen et al. [25] develop a framework for outsourcing secure computation that is similar to our protocol. However, their protocol requires specific properties in the underlying SMC protocol, where our protocol is designed to be truly generic. Our implementation and empirical performance analysis demonstrate that the added circuit overhead required by our protocol does not significantly affect the execution time for large circuits, and allows for truly generic SMC outsourcing. We examine the tradeoffs between these two protocols in Section 9 .

\section{Definitions of Security}

Outsourced two-party SMC protocols are designed to allow two parties of asymmetric computational capability to engage in a privacy-preserving computation with the assistance of an outsourcing party. We consider the situation where a mobile device possessing limited computational resources wishes to run an SMC protocol with an application server or other well-provisioned entity. To allow this, outsourcing protocols move the majority of the costly operations off of the mobile device and onto a Cloud provider without revealing to the Cloud either party's input or output to the computation. These protocols aim to provide security guarantees of privacy and correctness, and also attempt to minimize the computation required at the mobile device while still maintaining efficiency between the application server and the Cloud. To meet these goals in the outsourced setting, a number of careful security assumptions must be made.

\subsection{Two-party SMC security}

Our black box protocol is based on the execution of a two-party SMC protocol to obliviously compute the result. We make no assumptions about the techniques used or structure of this underlying protocol except that it meets the canonical definition of security against malicious adversaries using the ideal/real world paradigm [16]. Informally, this states that for any adversary participating in the two-party SMC protocol, there exists a simulator in an ideal world with a trusted third party running the computation where the output in both worlds is computationally indistinguishable. In this definition, the simulator in the ideal world is given oracle access to the adversary in the real world. Particularly in the two-party setting, there are a few caveats that must be assumed to make this definition feasible, and must be considered when designing an outsourced protocol that uses a two-party protocol in a black box manner.

First, it is known that two-party protocols cannot fully prevent early termination. In any execution, one party will receive their output of computation before the other party does. While certain techniques have been developed to partially solve this problem, there is no complete solution. While other outsourcing protocols have added in a fair-release guarantee, this guarantee comes at a cost. Either the protocol must provide additional commitments not guaranteed in a standard two-party protocol [8, 7], or the protocol must incorporate additional costly MAC operations to ensure the output is not tampered with [27, 38]. However, our black box protocol shows that if we treat the outsourced model like a standard two-party execution where fair release is not guaranteed, we can reduce the output consistency check to a single comparison on the mobile device. This allows the application server to recover its input 
first and potentially disrupt the mobile device's output, but mirrors the two-party execution guarantees exactly. Thus, our protocol optimizes execution overhead by not assuming a fair output release.

Second, it is possible that a malicious party can provide arbitrary input to the computation that may or may not correspond to their "real" input. While we cannot control what another party provides as input to the computation, this potential behavior must be handled by the definition of security. To handle this, the simulator in the ideal world, which has oracle access to the adversary in the real world, must not only be able to simulate the adversary's view of the protocol. Upon running the adversary with a given input, the simulator must also be able to recover the actual input used by the adversary. In our proofs of execution, the ideal world will invoke this simulator often as a mechanism to recover the adversary's input before initiating computation with the trusted third party. This ensures that the output in both worlds is indistinguishable.

Given these assumptions, a secure two-party SMC protocol provides two guarantees. The first is privacy, which means that a malicious adversary cannot learn anything about the other party's input or output value beyond what is revealed by his own output value. The second guarantee is correctness. This implies that even in the presence of a malicious adversary, the output of the protocol will be the correct output of the agreed upon function except with negligible probability.

For a formal definition of security and further discussion, refer to [16].

\subsection{Collusion assumptions}

Previous work in outsourcing secure multiparty computation makes careful assumptions about who in the computation is allowed to collude. Kamara et al. [27] discuss at length the theoretical justification for these assumptions. Essentially, to achieve an $n$-party outsourcing protocol with better complexity than a two-party SMC protocol, it must be assumed that the Cloud (i.e., the server aiding computation but not providing input to the function) cannot collude with any other party. Other outsourcing protocols have sought ways to relax this restriction without significantly increasing the complexity of the function being evaluated [8,7]. However, all of these protocols still assume that the application server and the Cloud cannot collude. We follow this assumption in our black box construction. As stated by Kamara et al., the existence of an outsourcing protocol where this particular collusion is allowed would imply an efficient two-party SMC scheme where one party performs work that is sub-linear with respect to the size of the function being evaluated. While there are techniques for such a two-party SMC protocol [15, 18], it is unclear that they can be applied to create such an outsourced protocol.

\subsection{Outsourced Security Definition}

We follow the security definition first established by Kamara et al. [27] but specified for the two-party scenario as in the work of Carter et al. [8, 7]. We slightly alter the definition to allow for the possibility of early termination by one of the parties, possibly preventing the other party from receiving output. We provide a summary of the definition here, and refer the reader to previous work for a complete discussion of the definition.

The real world setting is made up of three parties. Two of these parties provide input to the computation, while the third party takes on computational load for one of the two input parties. All three parties provide auxiliary random inputs to the protocol. Some subset of the three parties $A=\left(A_{1}, A_{2}, A_{3}\right)$ can behave maliciously, but we assume that the application server and the Cloud cannot collude. For the $i^{t h}$ honest party, $O U T_{i}$ is defined as its output, and for the $i^{\text {th }}$ corrupted party, $O U T_{i}$ is its view of the protocol. Then we define the $i^{\text {th }}$ partial output as:

$$
R E A L^{(i)}(k, x ; r)=\left\{O U T_{j}: j \in H\right\} \cup O U T_{i}
$$

Here, $k$ is the security parameter, $x$ is all inputs to the computed function, $r$ is the auxiliary randomness, and $H$ is the set of all honest parties.

The ideal world setting is made up of the same parties with the same inputs as the real world with the addition of a trusted third party that receives all parties' inputs, computes the desired function, and returns the output to all parties except the outsourced party that is not providing inputs to the function. Any party may abort the computation early or refuse to send input, in which case the trusted party sends no output. As in the standard two-party definition [16], it is possible for one party, upon receiving output from the trusted third party, to terminate the protocol, preventing the other party from receiving its output. For the $i^{\text {th }}$ honest party, $O U T_{i}$ is defined as its output received from the trusted party, and for the $i^{t h}$ corrupted party, $O U T_{i}$ is an arbitrary output value. Then we define the $i^{t h}$ partial output in the 


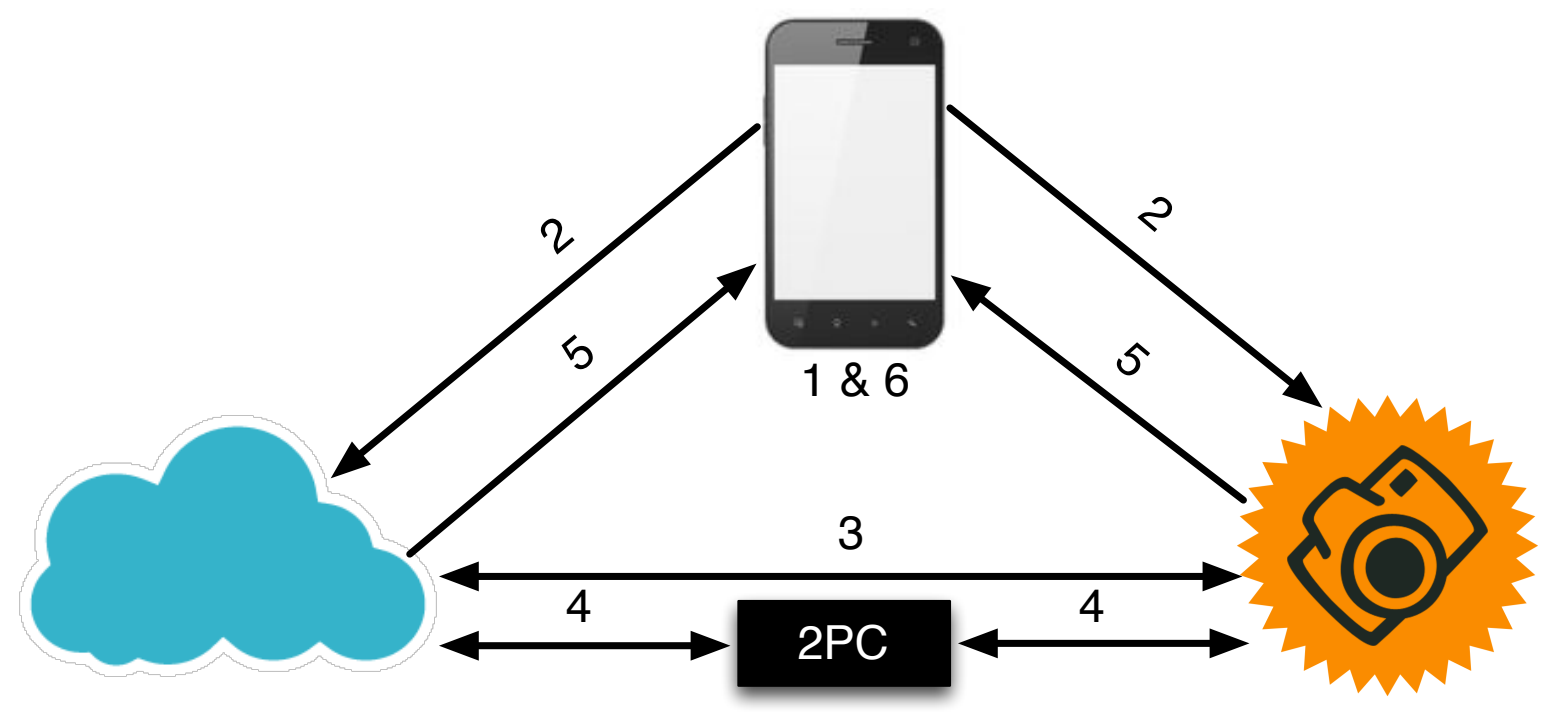

Figure 1: The complete black box outsourcing protocol. Note that the mobile device performs very little work compared to the application server and the Cloud, which execute a two-party SMC (2PC) protocol.

presence of independent malicious simulators $S=\left(S_{1}, S_{2}, S_{3}\right)$ as:

$$
I D E A L^{(i)}(k, x ; r)=\left\{O U T_{j}: j \in H\right\} \cup O U T_{i}
$$

Here, $k, x, r$, and $H$ are defined as above. In this real/ideal world setting, outsourced security is defined as follows:

Definition 1. An outsourcing protocol securely computes the function $f$ if there exists a set of probabilistic polynomialtime (PPT) simulators $\left\{\mathrm{Sim}_{1}, \mathrm{Sim}_{2}, \mathrm{Sim}_{3}\right\}$ such that for all PPT adversaries $\left(A_{1}, A_{2}, A_{3}\right)$, inputs $x$, and for all $i \in\{1,2,3\}:$

$$
\left\{R E A L^{(i)}(k, x ; r)\right\}_{k \in N} \stackrel{c}{\approx}\left\{I D E A L^{(i)}(k, x ; r)\right\}_{k \in N}
$$

Where $S=\left(S_{1}, S_{2}, S_{3}\right), S_{i}=\operatorname{Sim}_{i}\left(A_{i}\right)$, and $r$ is uniformly random.

\section{Protocol}

In this section, we formally define our black box outsourcing protocol. For a graphical representation, see Figure 1.

\subsection{Participants}

- SERVER: the application or web server participating in a secure computation with the mobile device. This party provides input to the function being evaluated.

- MobILE: the mobile device accessing SERVER to jointly compute some result. This party also provides input to the function being evaluated.

- Cloud a Cloud computation provider tasked with assisting Mobile in the expensive operations of the secure computation. This party executes a two-party SMC protocol in a black-box manner with SERVER, but does not provide an input to the function being evaluated. 


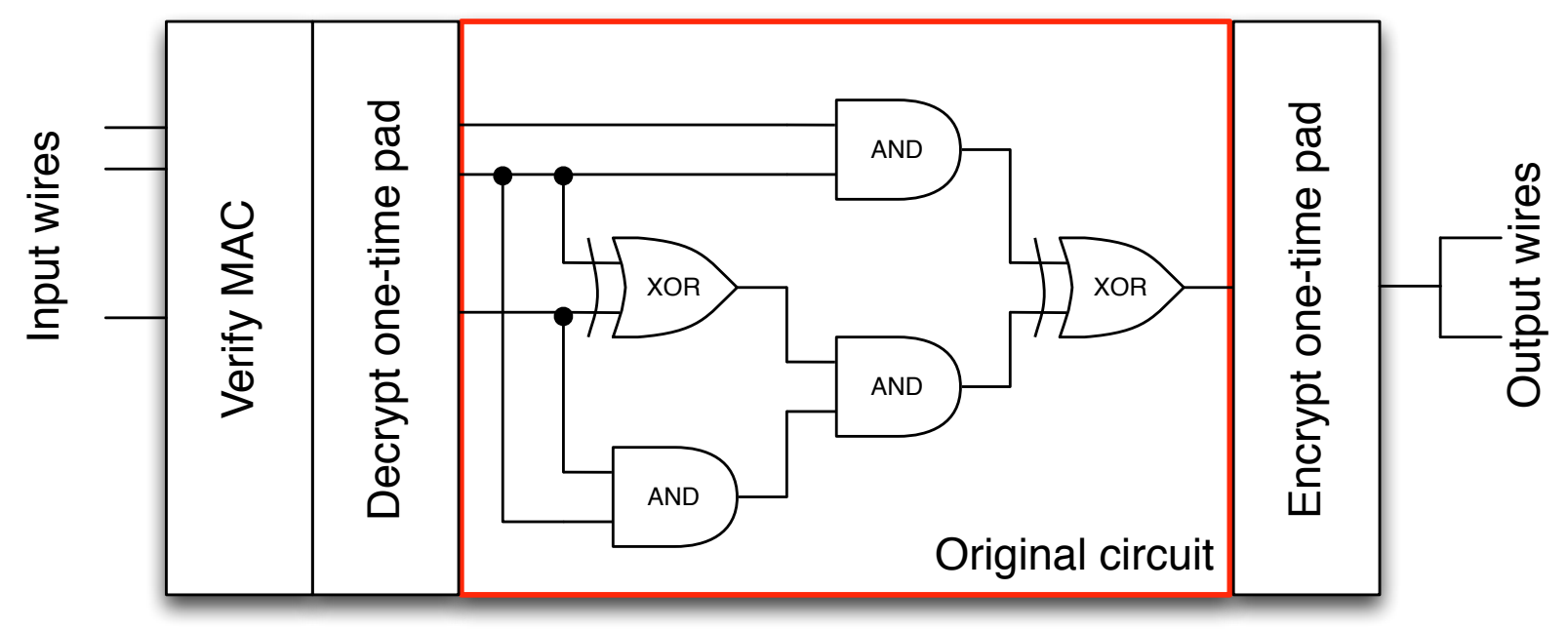

Figure 2: The process of augmenting a circuit for outsourcing. The original circuit is boxed in red. Essentially, we require that the mobile device's input be verified using a MAC and decrypted using a one-time pad before it is input into the function. After the result is computed, it must be re-encrypted using a one-time pad and delivered to both parties to guarantee that the mobile device will detect if either party tampers with the result.

\subsection{Overview}

The outsourcing protocol can be informally broken down as follows: first, the mobile device prepares its input by encrypting it and producing a MAC tag for verifying the input is not tampered with before it is entered into the computation. Since the application server and Cloud are assumed not to collude, one party receives the encrypted input, and the other party receives the decryption key. Both of these values are input into the secure two-party computation, and are verified within the secure two-party protocol using the associated MAC tags (see Figure 2). If the check fails, the protocol outputs a failure message. Otherwise, the second phase of the protocol, the actual evaluation of the SMC program, takes place. The third and final phase encrypts and outputs the mobile device's result to both parties, who in turn deliver these results back to the mobile device. Intuitively, since our security model assumes that the application server and the Cloud are never simultaneously malicious, at least one of these two will return the correct result to the mobile device. From this, the mobile will detect any tampering from the malicious party by a discrepancy in these returned values, eliminating the need for an output MAC. If no tampering is detected, the mobile device then decrypts the output of computation.

\subsection{Protocol}

Common Input: All parties agree on a computational security parameter $k$, a message authentication code (MAC) scheme $(\operatorname{Gen}(), \operatorname{Mac}(), \operatorname{Ver}())$, and a malicious secure two-party computation protocol $2 P C()$. All parties agree on a two-output function $f(x, y) \rightarrow f_{m}, f_{s}$ that is to be evaluated.

Private Input: MOBILE inputs $x$ while SERVER inputs $y$. We denote the bit length of a value as $|x|$ and concatenation as $x \| y$.

Output: SERVER receives $f_{s}$ and MOBILE receives $f_{m}$.

1. Input preparation: MoвILE generates a one-time pad $k_{f m}$ where $\left|k_{f m}\right|=\left|f_{m}\right|$. Mobile then generates two MAC keys $v_{s}=\operatorname{Gen}(k)$ and $v_{c}=\operatorname{Gen}(k)$. Finally, MoBILE generates a one-time pad $k_{m}$ where $\left|k_{m}\right|=$ $|x|+\left|k_{f m}\right|$. 


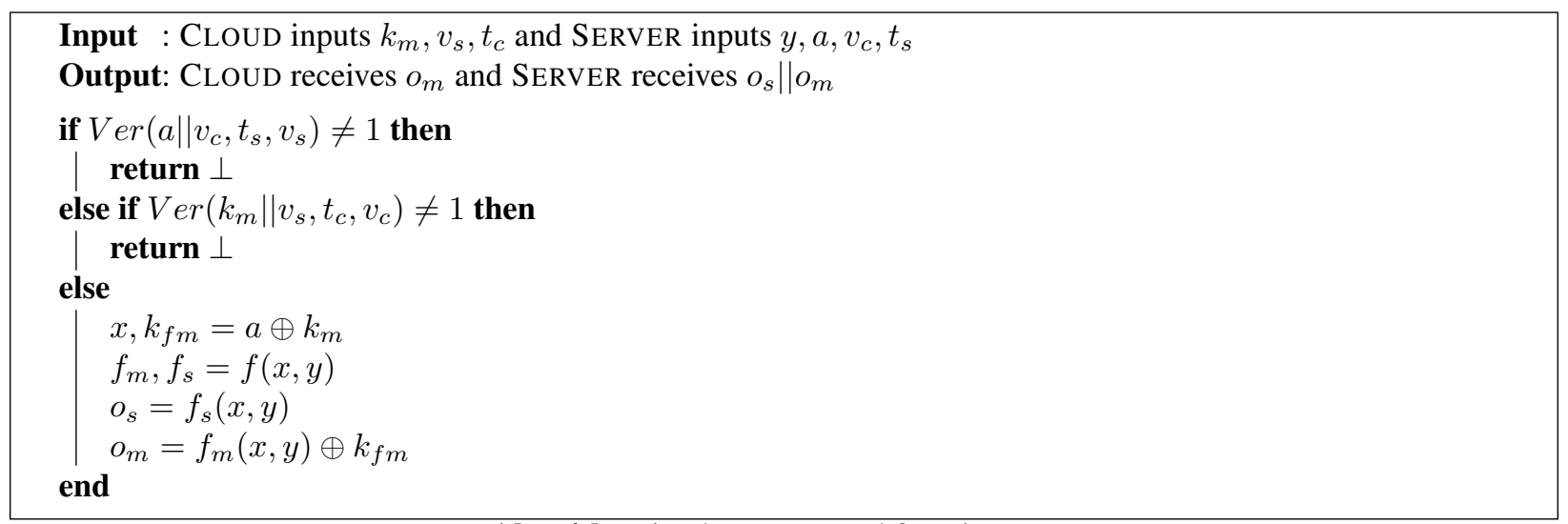

Algorithm 1: The augmented function

2. Input delivery: MobILE encrypts its input as $a=\left(x \| k_{f m}\right) \oplus k_{m}$. It then generates two tags $t_{s}=\operatorname{Mac}\left(a \| v_{c}, v_{s}\right)$ and $t_{c}=\operatorname{Mac}\left(k_{m} \| v_{s}, v_{c}\right)$. MoBILE delivers $a, v_{c}$, and $t_{s}$ to SERVER and $k_{m}, v_{s}$, and $t_{c}$ to CLOUD.

3. Augmenting the target function (Algorithm 1): All parties agree on the following augmented function $g\left(y, a, v_{c}, t_{s} ; k_{m}, v_{s}, t_{c}\right)$ to be run as a two-party SMC computation:

(a) If $\operatorname{Ver}\left(a \| v_{c}, t_{s}, v_{s}\right) \neq 1$ or $\operatorname{Ver}\left(k_{m} \| v_{s}, t_{c}, v_{c}\right) \neq 1$ output $\perp$.

(b) Set $x \| k_{f m}=a \oplus k_{m}$

(c) Run the desired function $f_{s}, f_{m}=f(x, y)$

(d) Set output values $o_{s}=f_{s}$ and $o_{m}=f_{m} \oplus k_{f m}$

(e) Output $o_{s} \| o_{m}$ to SERVER and $o_{m}$ to CLOUD

4. Two-party computation: SERVER and CLOUD execute a secure two-party computation protocol $2 P C\left(g() ; y, a, v_{c}, t_{s} ; k_{m}, v_{s}, t_{c}\right)$ evaluating the augmented function.

5. Output verification: CLOUd delivers its output from the two-party computation, $o_{m}$ to MobiLE. SERVER also delivers the second half of its output $o_{m}^{\prime}$ to MoBILE. MoBILE verifies that $o_{m}=o_{m}^{\prime}$.

6. Output recovery: SERVER receives output $f_{s}=o_{s}$ and MOBILE receives output $f_{m}=o_{m} \oplus k_{f m}$

\section{Security}

Our black box outsourcing protocol is secure under the following theorem satisfying the security definition from Section 3

Theorem 1. The black box outsourced two-party protocol securely computes a function $f(x, y)$ in the following two corruption scenarios: (1) Any one party is malicious and non-cooperative with respect to the rest of the parties; (2) The Cloud and the mobile device are malicious and colluding, while the application server is semi-honest.

Note that these scenarios correspond exactly with the corruption scenarios in [7], and that the previous protocols described in [27] and [8] are only secure in corruption scenario (1). We outline sketches of the security proof here, with a complete proof in Section 6

\subsection{Malicious Cloud or Application Server}

The main idea behind the security in these two settings is that for whichever party is corrupted, we can rely on the other party to behave semi-honestly. Based on the security of the underlying two-party protocol, this ensures both that the augmented functionality is correctly evaluated and that the mobile device will receive unmodified output from 
one of the parties. Thus, the MAC on the input and the comparison of the output values prevents either party from modifying the Mobile device's private values. Furthermore, unlike the dual execution model by Huang et al. [22] where the output comparison leaks one bit of input, our output comparison is composed of two copies of the mobile output produced from a single, malicious-secure execution of the augmented circuit. Because of this, any discrepancy in the comparison only reveals that either the Cloud or Application Server tampered with the output prior to delivering it to the mobile device.

In the ideal world, the simulator works roughly as follows: begin the black box protocol with random inputs. Then, invoke the simulator for the underlying two-party scheme $S_{2 P C}$ to recover the input of the malicious party and delivers that input to the trusted third party. Finally, $S_{2 P C}$ simulates the output $f(x, y)$. After running all consistency verifications, the simulator either sends an early termination signal to the trusted third party or completes the protocol normally.

\subsection{Malicious Mobile Device}

Because the mobile device simply provides MAC tagged input and receives its output after executing the two-party protocol, there is very little it can do to corrupt the computation besides providing invalid inputs that would simply cause the computation to terminate early. The simulator in this scenario accepts the mobile device's prepared inputs. Given both the Cloud and the Application Server's halves of the mobile device's input, the simulator can recover the necessary input by decrypting the one-time pad. If either of the MAC tags does not verify or if the mobile device terminates early, the simulator also terminates. Otherwise, it invokes the trusted third party to receive $f(x, y)$ and returns the result to the mobile device.

\subsection{Malicious Mobile Device and Cloud}

In this scenario, the security of our black box protocol simply reduces to the security of the underlying two-party scheme. The simulator in the ideal world accepts the input from the Mobile Device, then invokes the simulator of the underlying two-party SMC scheme $S_{2 P C}$ to recover the values input by the Cloud. Using these values combined with the values provided by the Mobile Device, the simulator can recover the Mobile input. If any of the verification checks within the augmented functionality fail, the simulator terminates. Otherwise, it delivers the recovered input to the trusted third party, and finishes $S_{2 P C}$ delivering the output of computation correctly formatted using the one-time pads recovered from the Cloud's input by $S_{2 P C}$.

\section{Proof of Security}

Here we provide the formal simulation proof of security for Theorem 1.

\subsection{Malicious MobILE $M^{*}$}

In the scenario where $M^{*}$ can adopt an arbitrary malicious strategy, we construct a simulator $S_{M}$ that, operating in the ideal world, can simulate $M^{*} s$ view of a real-world protocol execution and can recover $M^{*} s$ input for delivery to the trusted third party. We construct this simulator and prove it secure with the following hybrid of experiments.

$H y b 1^{(M)}(k, x ; r)$ : This experiment is identical to $R E A L^{(M)}(k, x ; r)$ except that the experiment uses the combination of $M^{*} s$ encrypted input $a$ and $k_{m}$ to recover the real input $x *$. It verifies the MAC tags $t_{s}$ and $t_{c}$ and aborts if either check fails.

Lemma 1. $R E A L^{(M)}(k, x ; r) \stackrel{c}{\approx} H y b 1^{(M)}(k, x ; r)$

Proof. Since the experiment is controlling both CLOUD and SERVER, it can simply decrypt the input $x^{*}$ using the key $k_{m}$. In addition, since the experiment holds both the verification keys, the protocol will terminate in both experiments if the MAC tags are incorrectly constructed.

$H y b 2^{(M)}(k, x ; r)$ : This experiment is identical to $H y b 1^{(M)}(k, x ; r)$ except that the experiment passes $x^{*}$ to the trusted third party, and returns the result $f\left(x^{*}, y\right) \oplus k_{f m}^{*}$ to $M^{*}$, where $k_{f m}^{*}$ is recovered in the previous hybrid. 
Lemma 2. $H y b 1^{(M)}(k, x ; r) \stackrel{c}{\approx} H y b 2^{(M)}(k, x ; r)$

Proof. Because both experiments use the input $x^{*}$ for computing the result, the output of the function in both worlds is indistinguishable. Furthermore, the recovered output key allows the experiment to present the result to $M^{*}$ exactly as it would be in a real world execution.

Lemma 3. $H y b 2^{(M)}(k, x ; r)$ runs in polynomial time.

Proof. This lemma follows trivially since a real world execution of the protocol runs in polynomial time and each intermediate hybrid adds only constant time operations.

We conclude the proof by letting $S_{M}$ execute $H y b 2^{(M)}(k, x ; r) . S_{M}$ runs $M^{*}$ and controls CLOUd and SERVER. $S_{M}$ terminates the ideal world execution if any consistency checks fail or if $M^{*}$ terminates at any point, and outputs whatever $M^{*}$ outputs at the end of the simulation. From Lemma 1 $3 . S_{M}$ proves Theorem 1 when MoBILE is malicious.

\subsection{Malicious SERVER $S^{*}$}

In the scenario where $S^{*}$ can adopt an arbitrary malicious strategy, we construct a simulator $S_{S}$ that, operating in the ideal world, can simulate $S^{*} s$ view of a real-world protocol execution and can recover $S^{*} s$ input for delivery to the trusted third party. We construct this simulator and prove it secure with the following hybrid of experiments.

$H y b 1^{(S)}(k, x ; r)$ : This experiment is identical to $R E A L^{(S)}(k, x ; r)$ except that the experiment prepares the MoBILE input according to the two-party protocol simulator $S_{2 P C}$ instead of using the real MOBILE input. It then prepares the new input according to the protocol and delivers the encrypted input and MAC tags to $S^{*}$.

Lemma 4. $R E A L^{(S)}(k, x ; r) \stackrel{c}{\approx} H y b 1^{(S)}(k, x ; r)$

Proof. Since the input is blinded by a one-time pad in both experiments, they are statistically indistinguishable.

$H y b 2^{(S)}(k, x ; r)$ : This experiment is identical to $H y b 1^{(S)}(k, x ; r)$ except that the experiment invokes the simulator of the two-party SMC protocol $S_{2 P C}$ instead of running the actual protocol. $S_{2 P C}$ is used to recover $S^{*} s$ actual input $y^{*}$. After recovering the full input, If $S^{*}$ tampers with MoBILE's input, $S_{2 P C}$ simulates $\perp$ and the experiment terminates. Otherwise, the experiment delivers $y^{*}$ to the trusted third party and simulates the output $f\left(x, y^{*}\right)$ concatenated with a random string $o_{r m}$.

Lemma 5. $H y b 1^{(S)}(k, x ; r) \stackrel{c}{\approx} H y b 2^{(S)}(k, x ; r)$

Proof. Based on the security definition of the underlying two-party SMC protocol, we know that a simulator exists that can simulate the protocol in a computationally indistinguishable way, as well as recover the input used by $S^{*}$. Based on the correctness guarantee of the two-party SMC protocol in conjunction with the unforgettability guarantee of the MAC protocol, it is computationally infeasible for $S^{*}$ to modify MoBILE's portion of the input. Finally, in both experiments the MOBILE output of the computation is blinded by a one-time pad, making the random output statistically indistinguishable from the real output.

$H y b 3^{(S)}(k, x ; r)$ : This experiment is identical to $H y b 2^{(S)}(k, x ; r)$ except that the experiment prevents the trusted third party from delivering input to the other party if $S^{*}$ modifies the MOBILE output $o_{r m}$ before returning it.

Lemma 6. $H y b 2^{(S)}(k, x ; r) \stackrel{c}{\approx} H y b 3^{(S)}(k, x ; r)$

Proof. Based on the correctness guarantee of the two-party SMC scheme and the fact that CLOUD is semi-honest in this scenario, then $S^{*}$ will be caught in either experiment, and early termination will be the result.

Lemma 7. $H y b 3^{(S)}(k, x ; r)$ runs in polynomial time.

Proof. This lemma follows trivially since a real world execution of the protocol runs in polynomial time, the simulator $S_{2 P C}$ runs in polynomial time, and all other intermediate hybrid adds only constant time operations.

We conclude the proof by letting $S_{S}$ execute $\operatorname{Hyb3}^{(S)}(k, x ; r) . S_{S}$ runs $S^{*}$ and controls CLOUd and MobiLE. $S_{S}$ terminates the ideal world execution if any consistency checks fail or if $S^{*}$ terminates at any point, and outputs whatever $S^{*}$ outputs at the end of the simulation. From Lemma 47, $S_{S}$ proves Theorem 1 when SERVER is malicious. 


\subsection{Malicious CLOUd $C^{*}$}

In the scenario where $C^{*}$ can adopt an arbitrary malicious strategy, we construct a simulator $S_{C}$ that, operating in the ideal world, can simulate $C^{*} s$ view of a real-world protocol execution and can recover $C^{*} s$ auxiliary input for delivery to the trusted third party. We construct this simulator and prove it secure with the following hybrid of experiments.

$H y b 1^{(C)}(k, x ; r)$ : This experiment is identical to $R E A L^{(C)}(k, x ; r)$ except that the experiment invokes the two-party SMC simulator $S_{2 P C}$, providing random inputs for SERVER and recovering $C^{*} s$ real input. Finally, simulate a random result $o_{r}$ at the end of the two-party computation.

Lemma 8. $R E A L^{(C)}(k, x ; r) \stackrel{c}{\approx} H y b 1^{(C)}(k, x ; r)$

Proof. Based on the security definition of the underlying two-party SMC protocol, we know that the simulator $S_{2 P C}$ can indistinguishably simulate the two-party execution and recover MOBILE's MAC tagged one-time pad as input by $C^{*}$. Because in both experiments the output of the circuit is blinded by a one-time pad, the outputs in both cases are statistically indistinguishable.

$H y b 2^{(C)}(k, x ; r)$ : This experiment is identical to $H y b 1^{(C)}(k, x ; r)$ except that if the experiment finds from the recovered input that $C^{*}$ modified the random key $k_{m}$, the experiment terminates.

Lemma 9. $H y b 1^{(C)}(k, x ; r) \stackrel{c}{\approx} H y b 2^{(C)}(k, x ; r)$

Proof. Based on the correctness guarantee of the two-party SMC scheme and the unforgettability of the MAC scheme, any change to $k_{m}$ will cause the circuit to output $\perp$, and will cause MOBILE to terminate except for a negligible probability. Thus, termination in both experiments in computationally indistinguishable.

$H y b 3^{(C)}(k, x ; r)$ : This experiment is identical to $H y b 2^{(C)}(k, x ; r)$ except that if the experiment aborts if $C^{*}$ modifies the output string $o_{r}$.

Lemma 10. $H y b 2^{(C)}(k, x ; r) \stackrel{c}{\approx} H y b 3^{(C)}(k, x ; r)$

Proof. Because SERVER is semi-honest and will not tamper with MOBILE's output, in both hybrids $C^{*}$ will be caught for tampering with the output and result in an abort of the protocol.

Lemma 11. $H y b 3^{(C)}(k, x ; r)$ runs in polynomial time.

Proof. This lemma follows trivially since a real world execution of the protocol runs in polynomial time, the simulator $S_{2 P C}$ runs in polynomial time, and all other intermediate hybrid adds only constant time operations.

We conclude the proof by letting $S_{C}$ execute $H y b 3^{(C)}(k, x ; r) . S_{C}$ runs $C^{*}$ and controls SERver and MobILE. $S_{C}$ terminates the ideal world execution if any consistency checks fail or if $C^{*}$ terminates at any point, and outputs whatever $C^{*}$ outputs at the end of the simulation. From Lemma 8 11, $S_{C}$ proves Theorem 1 when CLOUD is malicious.

\subsection{Malicious MobILe and CLOUd $M C^{*}$}

In the final scenario, the colluding parties $M C^{*}$ can adopt an arbitrary malicious strategy against SERVER. The simulator $S_{M C}$ that proves security in this scenario is essentially the two-party SMC simulator $S_{2 P C}$ with one small change. Rather than completely recovering $M C^{*} s$ input from the simulator, the experiment must combine the malicious MовILE input $a^{*}\left\|v_{s}^{*}\right\| t_{s}^{*}$ with the input recovered by $S_{2 P C}$ to learn the real input $x^{*}$ that is to be delivered to the trusted third party. Once this real input is retrieved, it simulates the result $f\left(x^{*}, y\right)$ exactly as $S_{2 P C}$ does. Since the added operations are constant time and $S_{2 P C}$ runs in polynomial time, we have that $S_{M C}$ proves Theorem 1 when both MobILE and CLOUd are malicious and colluding. Note that, as in the underlying two-party SMC scheme, this scenario does not guarantee that the output will be released fairly to SERVER. However, it does guarantee privacy and correctness of the output. 


\begin{tabular}{c|rrc|rrc}
\hline Program Name & SS13 Total & BB Total & Increase & SS13 Non-XOR & BB Non-XOR & Increase \\
\hline Dijkstra10 & 259,232 & 456,326 & $1.8 \mathrm{x}$ & 118,357 & 179,641 & $1.5 \mathrm{x}$ \\
\hline Dijkstra20 & $1,653,542$ & $1,949,820$ & $1.2 \mathrm{x}$ & 757,197 & 849,445 & $1.1 \mathrm{x}$ \\
\hline Dijkstra50 & $22,109,732$ & $22,605,018$ & $1.0 \mathrm{x}$ & $10,170,407$ & $10,324,317$ & $1.0 \mathrm{x}$ \\
\hline MatrixMult3x3 & 424,748 & $1,020,196$ & $2.4 \mathrm{x}$ & 161,237 & 345,417 & $2.1 \mathrm{x}$ \\
\hline MatrixMult5x5 & $1,968,452$ & $3,360,956$ & $1.7 \mathrm{x}$ & 746,977 & $1,176,981$ & $1.6 \mathrm{x}$ \\
\hline MatrixMult8x8 & $8,069,506$ & $11,354,394$ & $1.4 \mathrm{x}$ & $3,060,802$ & $4,075,082$ & $1.3 \mathrm{x}$ \\
\hline MatrixMult16x16 & $64,570,969$ & $77,423,481$ & $1.2 \mathrm{x}$ & $24,494,338$ & $28,458,635$ & $1.2 \mathrm{x}$ \\
\hline RSA128 & $116,083,727$ & $116,463,648$ & $1.0 \mathrm{x}$ & $41,082,205$ & $41,208,553$ & $1.0 \mathrm{x}$ \\
\hline
\end{tabular}

Table 1: A comparison of the original function size to the augmented outsourcing circuit. As the size of the original circuit grows, the increase in gates incurred by our outsourcing technique becomes vanishingly small.

\section{Performance Evaluation}

To demonstrate the practical efficiency of our black box outsourcing protocol, we implemented the protocol and examined the actual overhead incurred by the overhead operations. We initially considered comparing our black box protocol to existing implementations of outsourcing protocols [27, 8, 7]. However, these existing protocols are built on fixed underlying SMC techniques. As new protocols for two-party SMC are developed, the plug-and-play nature of our protocol allows for these new techniques to be applied, which would provide a different comparison for each underlying protocol. Instead, we chose to compare the overhead execution costs of our black box protocol to performing the same computation in the underlying two-party protocol. Because the mobile device computation requires seconds or less to execute, we focus our attention on the cost at the two executing servers. This performance analysis demonstrates two key benefits of our protocol. First, it gives a rough overhead cost for an entire class of two-party SMC protocols (in our case, garbled circuit protocols). Second, it allows us to demonstrate that our outsourcing technique allows a mobile device with restricted computational capability to participate in a privacy-preserving computation in approximately the same amount of time as the same computation performed between two servers. Essentially, we show that our protocol provides a mobile version of any two-party SMC protocol with nominal overhead cost to the servers. This is a novel evaluation methodology not used to evaluate previous black box SMC constructions, and provides a more intuitive estimate for performance when applying a new underlying SMC construction.

\subsection{System Design}

Our implementation of the black box outsourcing protocol uses the two-party garbled circuit protocol developed by shelat and Shen [44] as the underlying two-party SMC protocol. We selected this protocol because it is among the most recently developed garbled circuit protocols and it has the most stable public release. We emphasize that it is possible to implement our outsourcing on any two-party SMC protocol, such as the recent protocols developed to reduce the cost of cut-\&-choose [23, 32]. We implement our MAC within the augmented circuit using AES in cipherblock chaining mode (CBC-MAC), as the AES circuit is well-studied in the context of garbled circuit execution. This MAC implementation adds an invocation of AES per 128-bit block of input. Using the compiler developed by Kreuter et al. [31], the overhead non-XOR gate count in the augmented circuit based on input size is $\left(\frac{|x| 15686}{128}\right)$ for input $x$. We provide exact gate counts with overhead measurements for each tested application in Table 1 Our code will be made available upon publication.

\subsubsection{Testbed}

Our experiments were run on a single server equipped with 64 cores and 1 TB of RAM. For each execution, the application server and cloud were run as 32 processes communicating using the Message Passing Interface (MPI) framework. The mobile device used was a Samsung Galaxy Nexus with a $1.2 \mathrm{GHz}$ dual-core ARM Cortex-A9 processor and $1 \mathrm{~GB}$ of RAM, running Android version 4.0. The mobile device communicated with the test server over an $802.11 \mathrm{n}$ wireless connection in an isolated network environment. We ran each experiment 10 times and averaged the results, providing $95 \%$ confidence intervals in all figures. 


\begin{tabular}{ccc}
\hline Symmetric & Asymmetric & Communication (bits) \\
\hline 9 & 0 & $2(|x|+2 k)+4\left(\left|o_{m}\right|\right)$ \\
\hline
\end{tabular}

Table 2: The total operations and bandwidth required at the mobile device. Recall that $|x|$ is the length of the mobile input in bits, $k$ is the security parameter, and $\left|o_{m}\right|$ is the length of the mobile output in bits. When measured with the total protocol execution time, these operations are lost in the confidence intervals.

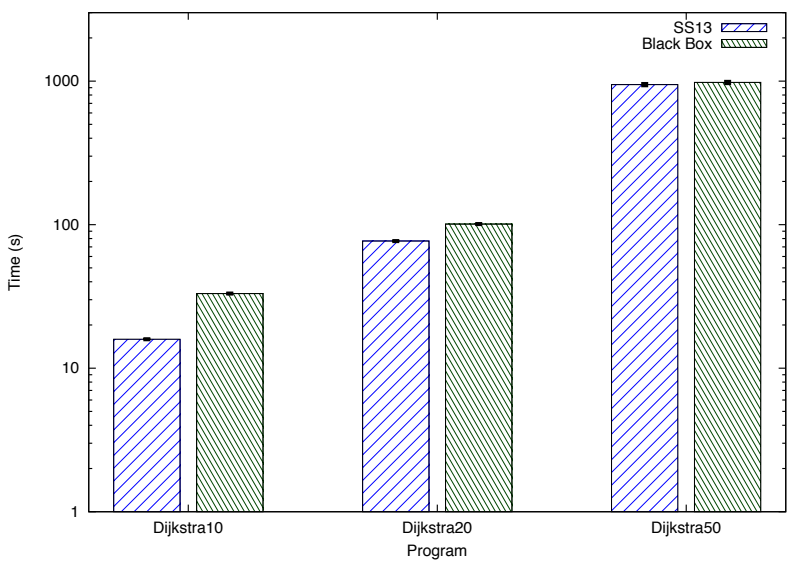

Figure 3: Dijkstra execution time in seconds for $k=80$. Note that for the largest input size, the execution overhead of outsourcing is almost non-existent.

\subsubsection{Test applications}

We selected a representative set of test applications from previous literature [7, 31, 44, 30] to examine the performance of our protocol over varying circuit and input sizes. We use all applications as implemented by Kreuter et al. [31] except for Dijkstra's algorithm, which was implemented by Carter et al. [8].

1. Dijkstra: this application accepts a weighted graph from one party and two node indices from the other party (i.e., start and end nodes), and calculates the shortest path through the graph from the start to the end node. We consider $n$-node graphs with 16 bit edge weights, 8 bit node identifiers, and a maximum degree of 4 for each node. We chose this problem as a representative application for the mobile platform.

2. Matrix Multiplication: this application accepts a matrix from both parties and outputs the matrix product. We consider this application for input size $n$, where each matrix is an $n \times n$ matrix of 32-bit integers. This test application demonstrates protocol behavior for increasing input sizes.

3. RSA: this application accepts a modulus $N$ and an exponent $e$ from one party, and a message $x$ from the other party, and computes the modular exponentiation $x^{e} \bmod N$. We consider input values where each value is 128 bits in length. While this is certainly too short for secure practical use, the size of the circuit provides a good benchmark for evaluating extremely large circuits.

\subsection{Execution Time}

With the mobile operations reduced to a minimal set, shown in Table 2, our experiments showed a diminishing cost of server overhead as the size of the test application increased. Considering Dijkstra's algorithm in Figure 3 shows that for a graph of 10 nodes, the outsourcing operations incur a $2.1 \mathrm{x}$ slowdown from running the protocol between two servers. However, as the number of graph nodes increases to 50, the confidence intervals for outsourced and server-only execution overlap, indicating a virtually non-existent overhead cost. When we compare these results to the gate counts shown in Table 1, we see that as the gate count for the underlying protocol increases, the additive cost of running the input MAC and output duplication amortize over the total execution time. This is to be expected from our predicted overhead of 15686 non-XOR gates for each CBC-MAC block in the input. However, since the mobile input for Dijkstra's algorithm is of a fixed size, we observe that increasing the application server input size does not add to the outsourcing overhead, showing the black box protocol to be more efficient for large circuit sizes with small mobile 


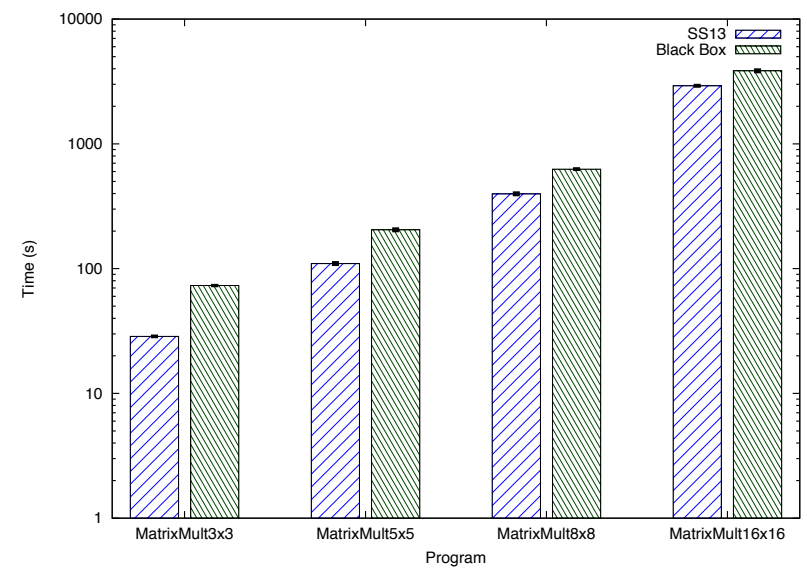

Figure 4: Matrix multiplication execution time in seconds for $k=80$. Note that the execution overhead still diminishes even as the mobile input size increases.

\begin{tabular}{c|c|c|r}
\hline Program Name & SS13 & BB & Increase \\
\hline Dijkstra10 & $16 \pm 1 \%$ & $33 \pm 1 \%$ & $2.1 \mathrm{x}$ \\
\hline Dijkstra20 & $77 \pm 1 \%$ & $100 \pm 1 \%$ & $1.3 \mathrm{x}$ \\
\hline Dijkstra50 & $940 \pm 2 \%$ & $980 \pm 2 \%$ & $1.0 \mathrm{x}$ \\
\hline MatrixMult3x3 & $28.6 \pm 0.8 \%$ & $73.2 \pm 0.5 \%$ & $2.6 \mathrm{x}$ \\
\hline MatrixMult5x5 & $110 \pm 2 \%$ & $200 \pm 2 \%$ & $1.9 \mathrm{x}$ \\
\hline MatrixMult8x8 & $400 \pm 2 \%$ & $627 \pm 0.9 \%$ & $1.6 \mathrm{x}$ \\
\hline MatrixMult16x16 & $2900 \pm 1 \%$ & $3800 \pm 2 \%$ & $1.3 \mathrm{x}$ \\
\hline RSA128 & $4700 \pm 2 \%$ & $4900 \pm 3 \%$ & $1.0 \mathrm{x}$ \\
\hline
\end{tabular}

Table 3: Comparing SS13 and Black Box runtime. All times in seconds. Note that as the circuit size increases, the increase in execution time caused by outsourcing becomes insignificant.

input.

When we consider a growing mobile input size, we observe the overhead cost of the MAC operation performed on the mobile input. In the matrix multiplication test program, we observed a $2.6 \mathrm{x}$ slowdown for the smallest input size of a $3 \times 3$ matrix (Figure 47. As in the previous experiment, this overhead diminished to a 1.3x slowdown for the largest input size, but diminished at a slower rate when compared to the circuit size. This is a result of additional AES invocations to handle the increasing mobile input size. However, the reduction in overhead shows that even as input sizes increase, the circuit size is still the main factor in amortizing overhead.

In our final experiment, we considered a massive circuit representing one of the most complex garbled circuit programs evaluated to date. When comparing the outsourced execution to a standard two-party execution, the overhead incurred by the outsourcing operations is almost non-existent, as shown in Table 3 This experiment confirms the trends of diminishing overhead cost observed in the previous two experiments. From this and previous work, we know that evaluating large circuits from mobile devices is not possible without outsourcing the bulk of computation. Given that many real-world applications will require on the order of billions of gates to evaluate, this experiment shows that our black box outsourcing technique allows mobile devices to participate in secure two-party computation at roughly the same efficiency as two server-class machines executing the same computation.

\subsection{Communication Cost}

Because transmitting data from a mobile device is costly in terms of time and power usage, we attempted to minimize the amount of bandwidth required from the mobile device. Thus, the bandwidth used by the mobile device for any given application can be represented as a simple formula, shown in Table 2 Because this bandwidth is nearly minimal and easily calculated for any test program, we focused our experimentation on examining the bandwidth overhead 


\begin{tabular}{c|c|c|r}
\hline Program Name & SS13 & \multicolumn{1}{|c|}{ BB } & Increase \\
\hline Dijkstra10 & $2.44 \times 10^{9}$ & $3.87 \times 10^{9}$ & $1.6 \mathrm{x}$ \\
\hline Dijkstra20 & $1.52 \times 10^{10}$ & $1.73 \times 10^{10}$ & $1.1 \mathrm{x}$ \\
\hline Dijkstra50 & $2.02 \times 10^{11}$ & $2.05 \times 10^{11}$ & $1.0 \mathrm{x}$ \\
\hline MatrixMult3x3 & $3.43 \times 10^{9}$ & $7.66 \times 10^{9}$ & $2.2 \mathrm{x}$ \\
\hline MatrixMult5x5 & $1.57 \times 10^{10}$ & $2.56 \times 10^{10}$ & $1.6 \mathrm{x}$ \\
\hline MatrixMult8x8 & $6.43 \times 10^{10}$ & $8.73 \times 10^{10}$ & $1.4 \mathrm{x}$ \\
\hline MatrixMult16x16 & $5.11 \times 10^{11}$ & $6.01 \times 10^{11}$ & $1.2 \mathrm{x}$ \\
\hline RSA128 & $8.69 \times 10^{11}$ & $8.72 \times 10^{11}$ & $1.0 \mathrm{x}$ \\
\hline
\end{tabular}

Table 4: Comparing SS13 and Black Box bandwidth usage between the parties performing the generation and evaluation of the garbled circuit. All bandwidth in bytes. Note that the size of the original circuit dominates the bandwidth required between the two servers. As this circuit grows in size, the overhead bandwidth required for outsourcing is amortized.

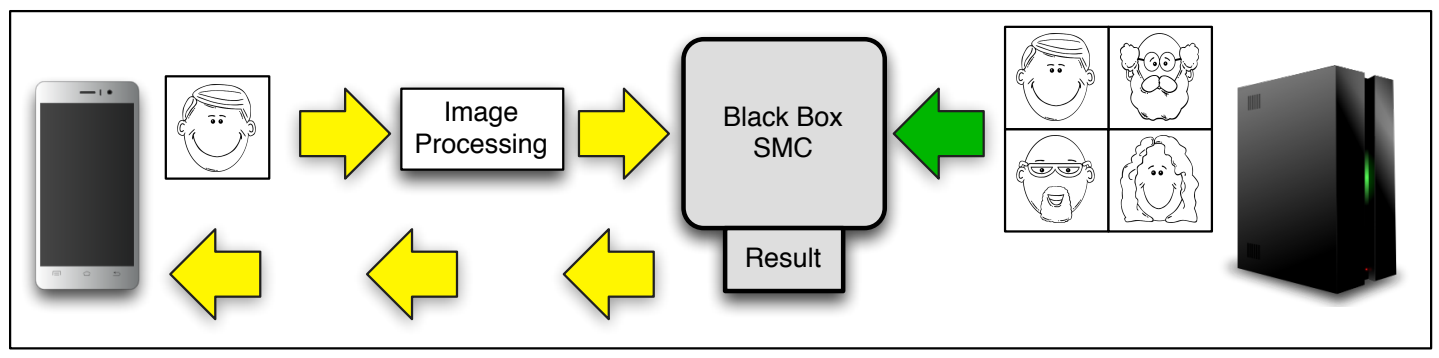

Figure 5: An example of the facial recognition application.

incurred between the application server and the Cloud.

As in the case of execution time, Table 4 shows an inverse relation between circuit size and overhead cost. Before running the experiment, we predicted that the bandwidth overhead would approximately match the overhead in circuit size shown in Table 1. The experiments confirmed that the actual bandwidth overhead was equal to or slightly larger than the overhead in non-XOR gates in the circuit. The reason for this correlation is twofold. First, the free-XOR technique used in the shelat-Shen protocol allows XOR gates to be represented without sending any data over the network. Thus, adding additional XOR gates does not incur bandwidth cost. Second, in cases where the actual overhead is slightly larger than the circuit size overhead, we determined that the added cost was a result of additional oblivious transfers. These operations require the transmission of large algebraic group elements, so the test circuits which incurred increased overhead from the growth of the mobile input showed a slightly larger bandwidth overhead as well. Ultimately, as in the case of execution time, our experiments demonstrate that the black box outsourcing scheme incurs minimal bandwidth usage at the mobile device with diminishing bandwidth overhead between the application server and the Cloud.

\section{Application: Facial Recognition}

The growing number of mobile applications available present a wealth of potential for applying privacy-preserving computation techniques to the mobile platform. Carter et al. [8] demonstrated one potential application with their privacy-preserving navigation app, and Mood et al. [38] presented a friend-finding application. We present a third mobile-specific application: facial recognition. In this setting, a secret operative or law enforcement agent carrying a mobile device needs to analyze a photo of a suspected criminal using an international crime database (see Figure 5 . The database, managed by an international organization, would compare the photo to their database in a privacypreserving manner, returning a match if the suspect appears in the database. In this scenario, the agent must keep the query data private to prevent insiders from learning who is being tracked, and the international organization must keep the database private from agents associated with any particular nation.

To implement this application, we use the facial recognition techniques developed for the Scifi protocol of Osadchy 


\begin{tabular}{cr}
\hline Program Name & Time \\
\hline FaceRec10 & $87.1 \pm 0.9 \%$ \\
\hline FaceRec100 & $170 \pm 2 \%$ \\
\hline FaceRec1000 & $1000 \pm 2 \%$ \\
\hline
\end{tabular}

Table 5: Runtime results showing the time it takes to determine what the input face is when a database of 10, 100, or 1000 faces is used. Time indicates the total runtime of the garbled circuit part of the computation. All time in seconds. These results demonstrate how outsourcing allows an application designed for desktop-class machines to be efficiently executed from the mobile platform.

et al. [41]. They develop a technique for two servers to perform efficient facial recognition using discrete parameters, which can more easily be manipulated in secure computation protocols. They combine machine learning techniques in a preprocessing phase with a secure online phase that compares the hamming distance of photos represented as bit strings. To demonstrate our application, we implement the online comparison phase of this protocol in our black box outsourcing protocol (the $F_{\text {threshold }}$ function in their work). The mobile device provides a 928 bit representation of a photo, while the application server provides a database of representations containing 10, 100, and 1000 faces.

Our results show that given a database of 10 faces, the outsourced protocol can run the online phase in approximately 87 seconds (see Table 5). As the size of the facial database increases, the execution time for comparing across the entire database grows. This growing cost is a result of the large cost of representing the facial database as garbled input. Provided with a two-party SMC protocol that more efficiently computes over large data sets, our black box protocol could be used to move this application from feasible to practical. This demonstrates that an application designed and implemented to run between two servers can be feasibly executed from a mobile device. As new, more heavyweight applications are developed, our technique for outsourcing allows any of those applications to be executed from a mobile device with comparable efficiency to the server platform.

\section{Comparison to Previous Techniques}

While our implementation and evaluation in the previous section represents the first empirical analysis of black box outsourcing, two other protocols have been proposed in the literature, which we term KMR [27] and JNO [25]. We evaluate the tradeoffs between each technique in this section.

\subsection{KMR}

While the main focus of their work is the fixed outsourcing protocol Salus [27], Kamara et al. sketch a black box technique for outsourcing any two-party computation protocol. Essentially, their protocol encodes each bit of the MoвILE input as a bit string of length $k$ for some computational security parameter $k$. This encoded input, along with the mappings for reversing this encoding, is secret shared between SERVER and CLOUD, and then restored using only XOR gates inside the circuit. A similar encoding technique is used to maintain both privacy and integrity of the output from the circuit. This technique has the advantage of adding only XOR gates to the circuit, which can be transmitted and evaluated cheaply using many SMC techniques. However, it also requires that the mobile input and output be expanded by a factor of $k$. By contrast, our evaluation demonstrates that the overhead caused by adding AND gates to the computation is minimal, and the MoBILE bandwidth use is kept to $O(|x|)$ with a small constant multiple. This is particularly advantageous on smartphones, where data usage is often restricted by slow network speeds or provider-imposed bandwidth caps.

\subsection{JNO}

Developed concurrently to our protocol, Jakobsen et al. [25] presented a framework for outsourcing SMC protocols across any number of "worker" servers. Their protocol follows a similar procedure to our own, but they describe a novel MAC construction that allows the MOBILE input to be checked using only inexpensive linear operations in the 
circuit. Essentially, their technique requires that the MAC key be committed at the start of the protocol, then opened once the rest of the input values are committed to the computation. Once the key is opened, it can be multiplied as a known constant with the MOBILE input, which is secret shared according to the underlying SMC protocol. A simple multiplicative MAC can then be verified before computation continues. The advantage of this scheme is that it does not incur the $k$ factor expansion of KMR while still adding only linear operations to the underlying circuit (e.g., XOR for boolean circuits). The tradeoff is that the underlying SMC protocol must allow for reactive computation (i.e., private values can be opened in the middle of computation). While this property is common in secret-sharing SMC protocols, it is difficult to achieve with garbled circuits. The generic technique for making garbled circuits into a reactive SMC protocol requires additional, MAC operations inside the circuit [19]. More efficient reactive garbled circuit protocols exist [14, 38], but require special constructions that cannot be combined with all garbled circuit protocols in a generic way. Our protocol allows for true black box outsourcing of any SMC protocol (reactive or non-reactive), and our empirical performance evaluation demonstrates that the overhead of adding AND operations to the circuit is minimal when the circuit size is large. This setting is preferable for computation that is more efficiently evaluated using garbled circuits than arithmetic secret-sharing SMC schemes.

\section{Conclusion}

The growing popularity of the mobile platform is creating a strong need for privacy-preserving computation in mobile applications. However, as most SMC techniques currently require significant processing and bandwidth resources, secure outsourcing protocols have been developed to assist mobile devices in performing the most expensive cryptographic operations associated with these protocols. In this work, we develop a technique for outsourcing any two-party SMC protocol in a black box manner. Our protocol securely offloads the cost of the SMC protocol to the Cloud, providing maximal efficiency to the mobile device while maintaining strong security guarantees. Our performance evaluation shows that as the complexity of the program being evaluated increases, the cost of outsourcing diminishes. As a result, we enable execution of any SMC protocol from a mobile device at approximately the same efficiency as running the protocol between two servers.

\section{References}

[1] G. Asharov, Y. Lindell, T. Schneider, and M. Zohner. More efficient oblivious transfer and extensions for faster secure computation. In Proceedings of the 2013 ACM SIGSAC Conference on Computer \& Communications Security, 2013.

[2] M. J. Atallah and K. B. Frikken. Securely outsourcing linear algebra computations. In Proceedings of the ACM Symposium on Information, Computer and Communications Security (ASIACCS), 2010.

[3] D. Beaver. Server-assisted cryptography. In Proceedings of the workshop on New security paradigms (NSPW), 1998.

[4] M. Bellare, V. T. Hoang, and P. Rogaway. Foundations of garbled circuits. In Proceedings of the ACM Conference on Computer and Communications Security, 2012.

[5] M. Blanton, A. Steele, and M. Alisagari. Data-oblivious graph algorithms for secure computation and outsourcing. In Proceedings of the ACM SIGSAC Symposium on Information, Computer and Communications Security, 2013.

[6] H. Carter, C. Amrutkar, I. Dacosta, and P. Traynor. For your phone only: custom protocols for efficient secure function evaluation on mobile devices. Journal of Security and Communication Networks (SCN), 7(7):1165-1176, 2014.

[7] H. Carter, C. Lever, and P. Traynor. Whitewash: Outsourcing Garbled Circuit Generation for Mobile Devices. In Proceedings of the Annual Computer Security Applications Conference (ACSAC), 2014.

[8] H. Carter, B. Mood, P. Traynor, and K. Butler. Secure Outsourced Garbled Circuit Evaluation for Mobile Devices. In Proceedings of the USENIX Security Symposium, 2013.

[9] H. Carter, B. Mood, P. Traynor, and K. Butler. Outsourcing secure two-party computation as a black box (short paper). In Proceedings of the International Conference on Cryptology and Network Security (CANS), 2015.

[10] I. Damgård, M. Keller, E. Larraia, V. Pastro, P. Scholl, and N. P. Smart. Practical covertly secure mpc for dishonest majority or: Breaking the spdz limits. In Computer Security-ESORICS, 2013.

[11] I. Damgård, V. Pastro, N. Smart, and S. Zakarias. Multiparty computation from somewhat homomorphic encryption. In Advances in Cryptology-CRYPTO, 2012.

[12] D. Demmler, T. Schneider, and M. Zohner. Ad-hoc secure two-party computation on mobile devices using hardware tokens. In Proceedings of the USENIX Security Symposium, 2014.

[13] T. K. Frederiksen, T. P. Jakobsen, J. B. Nielsen, P. S. Nordholt, and C. Orlandi. Minilego: Efficient secure two-party computation from general assumptions. In Advances in Cryptology-EUROCRYPT, 2013. 
[14] T. K. Frederiksen and J. B. Nielsen. Fast and maliciously secure two-party computation using the gpu. In Applied Cryptography and Network Security, 2013.

[15] C. Gentry. A fully homomorphic encryption scheme. PhD thesis, Stanford University, 2009.

[16] O. Goldreich. Foundations of Cryptography: Volume 2 Basic Applications. Cambridge Univ. Press, 2004.

[17] O. Goldreich, S. Micali, and A. Wigderson. How to play any mental game. In Proceedings of the Annual ACM Symposium on Theory of Computing, 1987.

[18] S. D. Gordon, J. Katz, V. Kolesnikov, A.-1. B. Labs, F. Krell, and M. Raykova. Secure Two-Party Computation in Sublinear (Amortized) Time. In Proceedings of the ACM conference on Computer and communications security (CCS), 2012.

[19] C. Hazay and Y. Lindell. Efficient Secure Two-Party Protocols: Techniques and Constructions. Springer-Verlag, 2010.

[20] Y. Huang, P. Chapman, and D. Evans. Privacy-preserving applications on smartphones. In Proceedings of the USENIX Workshop on Hot Topics in Security, 2011.

[21] Y. Huang, D. Evans, J. Katz, and L. Malka. Faster Secure Two-Party Computation Using Garbled Circuits. In Proceedings of the USENIX Security Symposium, 2011.

[22] Y. Huang, J. Katz, and D. Evans. Quid-pro-quo-tocols: Strengthening semi-honest protocols with dual execution. In Proceedings of the IEEE Symposium on Security and Privacy, 2012.

[23] Y. Huang, J. Katz, and D. Evans. Efficient secure two-party computation using symmetric cut-and-choose. In Advances in CryptologyCRYPTO, 2013.

[24] Y. Huang, J. Katz, and V. Kolesnikov. Amortizing garbled circuits. In Advances in Cryptology-CRYPTO, 2014.

[25] T. P. Jakobsen, J. B. Nielsen, and C. Orlandi. A framework for outsourcing of secure computation. In Proceedings of the ACM Workshop on Cloud Computing Security (CCSW), 2014.

[26] S. Kamara, P. Mohassel, and M. Raykova. Outsourcing multi-party computation. Cryptology ePrint Archive, Report 2011/272, 2011. http://eprint.iacr.org/

[27] S. Kamara, P. Mohassel, and B. Riva. Salus: A system for server-aided secure function evaluation. In Proceedings of the ACM conference on Computer and communications security (CCS), 2012.

[28] L. Kelion. Apple toughens icloud security after celebrity breach. http://www.bbc.com/news/technology-29237469 2014.

[29] F. Kerschbaum. Collusion-resistant outsourcing of private set intersection. In Proceedings of the ACM Symposium on Applied Computing, 2012.

[30] B. Kreuter, a. shelat, B. Mood, and K. Butler. PCF: A portable circuit format for scalable two-party secure computation. In Proceedings of the USENIX Security Symposium, 2013.

[31] B. Kreuter, a. shelat, and C. Shen. Billion-Gate Secure Computation with Malicious Adversaries. In Proceedings of the USENIX Security Symposium, 2012.

[32] Y. Lindell. Fast cut-and-choose based protocols for malicious and covert adversaries. In Advances in Cryptology-CRYPTO, 2013.

[33] Y. Lindell and B. Pinkas. An efficient protocol for secure two-party computation in the presence of malicious adversaries. In Proceedings of the annual international conference on Advances in Cryptology, 2007.

[34] Y. Lindell and B. Pinkas. Secure two-party computation via cut-and-choose oblivious transfer. In Proceedings of the conference on Theory of cryptography, 2011.

[35] Y. Lindell and B. Riva. Cut-and-Choose Yao-Based Secure Computation in the Online/Offline and Batch Settings. In Advances in Cryptology?CRYPTO, 2014.

[36] D. Malkhi, N. Nisan, B. Pinkas, and Y. Sella. Fairplay-a secure two-party computation system. In Proceedings of the USENIX Security Symposium, 2004.

[37] P. Mohassel and B. Riva. Garbled circuits checking garbled circuits: More efficient and secure two-party computation. In Advances in Cryptology-CRYPTO, 2013.

[38] B. Mood, D. Gupta, K. Butler, and J. Feigenbaum. Reuse it or lose it: More efficient secure computation through reuse of encrypted values. In Proceedings of the ACM conference on Computer and communications security (CCS), 2014.

[39] B. Mood, L. Letaw, and K. Butler. Memory-efficient garbled circuit generation for mobile devices. In Proceedings of the IFCA International Conference on Financial Cryptography and Data Security (FC), 2012.

[40] J. B. Nielsen, P. S. Nordholt, C. Orlandi, and S. S. Burra. A new approach to practical active-secure two-party computation. In Advances in Cryptology-CRYPTO, 2012.

[41] M. Osadchy, B. Pinkas, A. Jarrous, and B. Moskovich. Scifi-a system for secure face identification. In Proceedings of the IEEE Symposium on Security \& Privacy, 2010.

[42] B. Pinkas, T. Schneider, N. P. Smart, and S. Williams. Secure two-party computation is practical. In Advances in Cryptology-ASIACRYPT, 2009.

[43] a. shelat and C.-H. Shen. Two-output secure computation with malicious adversaries. In Proceedings of the Annual international conference on Theory and applications of cryptographic techniques, 2011.

[44] a. shelat and C.-H. Shen. Fast two-party secure computation with minimal assumptions. In Proceedings of the ACM conference on Computer and communications security (CCS), 2013.

[45] А. C. Yao. Protocols for secure computations. In Proceedings of the Annual Symposium on Foundations of Computer Science, 1982. 